\section{Traumatic gastric rupture following blunt abdominal trauma: a case report about an atypical modality of presentation}

\section{Dario Giambelluca, ${ }^{1}$ Dario Picone, ${ }^{1}$ \\ Natalino Carmelo Pennisi, \\ Bruno Luciani, ${ }^{3}$ Giuseppe Lo Re, Placido Romeo ${ }^{3}$ \\ 'Department of Radiology, University Hospital Paolo Giaccone, Palermo, Italy; 2Department of Radiology, University Hospital Gaetano Martino, Messina, Italy; ${ }^{3}$ Department of Radiology, San Vincenzo Hospital, Taormina (ME), Italy}

\begin{abstract}
Gastric rupture following blunt abdominal trauma is a rare presentation with a reported incidence of $0.02-1.7 \%$ in current literature. Traumatic gastric rupture is usually associated with other visceral injuries, such as splenic lesions and fractures. Prompt diagnosis and early intervention reduce mortality and morbidity. History of a recent meal has been implicated in traumatic gastric rupture. We report a case of blunt abdominal trauma with an isolated gastric rupture after a motor vehicle accident, managed successfully without any post-operative morbidity and mortality.
\end{abstract}

\section{Introduction}

Blunt abdominal trauma is a leading cause of morbidity and mortality amongst all ages. It commonly occurs due to motor vehicle collisions. Other causes include falls from height, seat-belt injuries and vigorous resuscitation. The incidence of "hollow viscus injuries" following blunt abdominal trauma varies from 4 to $15 \%$. Blunt gastric injuries are even rarer, occurring only in $0.02-1.7 \%$ of patients with blunt abdominal trauma. ${ }^{1}$ Isolated blunt gastric rupture is extremely rare, being normally associated with other intra and extra abdominal injuries, like splenic injury and fractures. ${ }^{1}$ Albeit uncommon, hollow viscus injuries after blunt trauma can have serious consequences if the diagnosis is missed or delayed. Mortality rate increases in parallel with timing of operative intervention $(<8$ hrs: $2 \%$; 8-16 hrs: 9\%; 16-25 hrs: 17\%; >24 hrs: $31 \%$ ) as well as the complication rates. ${ }^{2}$ We report a case of traumatic gastric rupture diagnosed and treated with good results.

\section{Case Report}

A 20-year-old male was admitted to the emergency room about half an hour after a road traffic accident, conscious and complaining of chest pain. He was riding a two wheeler as a pillion when he had a side impact collision with a car. There were no apparent external injuries except for a small bruise in epigastrium. He had not had a recent meal before trauma. There was no history of loss of consciousness, nausea or vomiting.

On physical examination, the patient appeared conscious and well oriented. He was hemodynamically stable with blood pressure at $110 / 60 \mathrm{mmHg}$ and pulse rate $100 / \mathrm{min}$, regular and of good volume. Temperature was $36.2{ }^{\circ} \mathrm{C}$. Respiratory rate was $20 /$ min with $\mathrm{SpO}^{2}$ of $95 \%$.

The abdomen was soft without tenderness. FAST examination was immediately performed, but subcutaneous emphysema represented an obstacle to medium abdomen exploration.

Plain X-ray of chest and abdomen did not show direct and indirect pathological signs, except for a left-sided pleural effusion. Contrast enhanced Computed Tomography (CECT) of Chest and Abdomen revealed a very small amount of free air in abdomen, a suspected renal artery bleeding and under-cuticular abdominal wall emphysema (Figure 1A). Detection of free intraperitoneal air, albeit of small volume, raised suspicion of a perforated hollow viscus, even though the exact location of the damage has not been demonstrated.

The patient was subsequently admitted to the General Surgery Department. Hematological and biochemical parameters were within normal limit. Hematocrit was completely stable and patient repeated the CECT after 3 hours without demonstrating substantial modifications.

About 14 hours later, the patient suddenly complained of tenderness and guarding of upper abdomen. There was also an increased white blood cell count. A new CECT examination was performed, revealing clear signs of a gastric lesion with extensive pneumoperitoneum and free fluid collection within abdomen (Figure 1B).

The patient was taken up for exploratory laparotomy as an emergency case, confirming the presence of a full thickness gastric rupture, which was about $2 \mathrm{~cm}$ in length, located on the anterior-lateral surface of the stomach, 1 centimeter above the cardia.

There were associated several other findings, such as renal contusions, traumatic epigastric omental hernia with air trapping, subcutaneous emphysema and linea
Correspondence: Giuseppe Lo Re, Department of Radiology, University Hospital Paolo Giaccone, via del Vespro, 129, 90127 Palermo, Italy.

Tel: +39.091.6552316 - Fax: +39.091.6552324.

E-mail: giuseppe.lore12@gmail.com

Key words: Gastric rupture; Blunt trauma; Abdomen; Abdominal trauma.

Acknowledgements: I would like to thank Fabio Vasta, Giuseppe Leonardi, Rosario Blandino, Giacomo Filoni, Lamberto Salvo and Maria Cristina Inserra for their valuable advice and comments on this manuscript.

Contributions: DG made substantial contributions to conception, design and in drafting the article. DP and NCP participated in revising it critically for important intellectual content. $\mathrm{BL}$ interpreted the radiologic examination findings and the patient data regarding the differential diagnosis of the disease. GLR made substantial contributions to acquisition, analysis and interpretation of data. PR designed and reviewed the manuscript and gave final approval of the version to be submitted. All authors read and approved the final manuscript.

Conflict of interest: the authors declare no potential conflict of interest.

Note: informed consent to publish could not be obtained from the patient or their guardian/next of kin in this instance, despite exhaustive attempts have been made to contact the patient and guardian/next of kin, including but not limited to: multiple attempts over a period of 3 months to contact the patient/family/next of kin by phone, email and letter; following up with referring center/other care providers for more information on patient/family/next of kin whereabouts and contact details; research online/with local authorities to obtain patient whereabouts and contact details. In addition, we can confirm that this case has been sufficiently anonymized to protect patient's identity in the manuscript.

Received for publication: 1 February 2017. Revision received: 19 April 2017.

Accepted for publication: 19 April 2017.

This work is licensed under a Creative Commons Attribution 4.0 License (by-nc 4.0).

CCopyright D. Giambelluca et al., 2017

Licensee PAGEPress, Italy

Emergency Care Journal 2017; 13:6609 doi:10.4081/ecj.2017.6609

alba tearing. A two-layer closure was performed for gastric rupture (Figure 2) followed by a thorough peritoneal lavage. Post-operative recovery was uneventful.

An X-ray examination, with administra- 


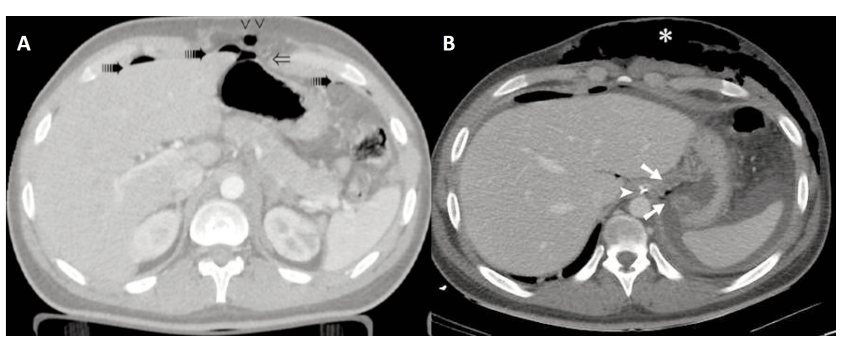

Figure 1. Twenty-year-old male with gastric rupture following blunt abdominal trauma. Technique: computed tomography (CT) scans $(140 \mathrm{kV}$, modulated $\mathrm{mA}, 6 \mathrm{~mm}$ slice thickness, 110 $\mathrm{mL}$ of Iomeron 400, portal venous phase) of abdomen. Findings: A) Omental traumatic epigastric hernia in the first CT exam (lung window). The finding of vessels within mesenteric fat suggests probable omental origin (empty arrow). Air trapping in the traumatic hernia (arrowheads). There are also some intra-peritoneal air bubbles (discontinuous arrows). B) Follow-up CT scan obtained 24 hours later shows a tear below the cardia (arrows). Nasogastric catheter (arrowhead). Edema of diaphragmatic crura is less evident compared to the first CT exam (not showed). Subcutaneous emphysema (asterisk).

tion of oral contrast agent, was performed 10 days after surgery, ensuring full recovery of the patient, who started to eat without issues. At 2-month follow-up, he was asymptomatic on clinical examination.

\section{Discussion}

Gastric rupture following blunt abdominal trauma is a rare entity. It usually results from motor vehicle collisions, assaults, recreational accidents or falls. The most commonly injured organs are spleen, liver, retroperitoneal organs, small bowel, kidneys, bladder, colorectum, diaphragm and pancreas. Physical examination findings are notoriously unreliable for several reasons (e.g. distracting injuries, altered mental state and drug and alcohol addiction). Men are slightly more often involved than women.

Usually, stomach is not injured in blunt trauma setting, because it is relatively mobile, provided with thick walls and protected by rib cage; however, if distended by food, liquid or gas, stomach is more easily damaged. Traumatic gastrointestinal lesions can be caused by three different injury mechanisms: i) crush injury, which occurs when an organ is compressed violently against the spine by directly applied pressure; ii) burst injury, which occurs when a sudden, violent compression of the abdominal wall is applied to a filled and extended hollow viscous, without any direct mechanical compression, resulting in an increase of intraluminal pressure; history of a recent meal has been often observed; iii) shear injury, caused by sudden deceleration of an organ at one point of fixation or its vascular pedicles. $^{3}$

Gastric rupture following Heimlich maneuver, bag and mask ventilation and cardiopulmonary resuscitation has also been reported in literature. ${ }^{4}$

Actually, the most commonly used injury scoring grades for traumatic injuries of stomach is The American Association for the Surgery of Trauma injury scoring scale: according to this score, increasing morbidity and mortality correlates with increasing severity of injury, which is assessed on the basis of depth of parenchymal damage and involvement of vessels. ${ }^{5}$ Partial rupture of the stomach wall may lead to full thickness rupture at a later stage. The anterior gastric wall is the most common site of rupture, followed by greater curvature and posterior wall.

Gastric rupture is frequently associated with adjacent solid organs injuries and bone fractures. In literature, lung injury and pneumothorax or hemothorax have been reported up to $83 \%-93 \%$ of cases. ${ }^{6}$ Septic complications, like abscess and gastric fistulae, are directly related to massive intraperitoneal contamination and represent most relevant issues.

Free intra-peritoneal air following a gastric tear can be easily detected on plain abdomen and chest radiographs, though this finding is found only in $66 \%$ of cases.

Computed Tomography (CT) is the gold standard examination for early diagnosis of gastric perforation, decreasing the risk of secondary complications like peritoneal contamination, sepsis and shock. CT results more useful for visualization of intraabdominal free air and detection of associated solid organ injuries.

The characteristic CT findings suggestive of gastric rupture include: free subdiaphragmatic air, accentuated visualization of an outlined falciform ligament (falciform ligament sign), intra-peritoneal nasogastric tube dislocation and abrupt wall thickening associated with adjacent dirty fat density. ${ }^{7}$ Our patient did not show any of these signs either at first or second CECT examinations.

Intra-peritoneal air bubbles should rise suspicion of a very little visceral injury partially covered by omentum; in our case, gastric lesion became evident only after some hours, due to a better contrast enhancement of the traumatic parietal necrosis of stomach.

In our opinion, parietal vessels distraction should be invoked as a reasonable cause for the damage (shear injury mechanism). This modality explains exhaustively the finding of edema into diaphragmatic crura. The atypical site of the gastric lesion in our patient could be explained by stomach emptiness.

Traumatic omental hernia was suspected because of the presence of linea alba tearing and it should be responsible of subcutaneous emphysema and air trapping in non-dependent part of abdomen, in continuity with intra-peritoneal space.

Our patient did not show any complications in post-surgical follow-up. 


\section{Conclusions}

Traumatic gastric rupture represents a challenging diagnosis in absence of other suggestive findings of wall tears. Even in asymptomatic patients, the presence of a small amount of free air in abdomen after blunt trauma should raise suspicion of a visceral intra-peritoneal injury. Short-term follow-up is mandatory to plan an appropriate surgical strategy and avoid complication occurrence.

Subcutaneous emphysema without pneumothorax in patients with omental hernia should be explained by a tearing of linea alba.

\section{References}

1. Tejerina Alvarez EE, Holanda MS, López-Espadas F, et al. Gastric rupture from blunt abdominal trauma. Injury 2004;35:228-31.

2. Weinberf JA, Fabian TC. Jnjures to the stomach, small bowel, colon and rectum. Trauma Thermal Injury 2005;7;18.

3. Ritesh M, Ashutosh S, Pankaj M. Gastric rupture following blunt trauma abdomen: a case report. Indian J Clin Pract 2013;23:859-60.

4. Haslam N, Campbell GC, Duggan JE. Gastric rupture associated with the use of the laryngeal mask airway during cardiopulmonary resuscitation. BMJ 2004;329:1225-6.

5. Moore EE, Cogbill TH, Malangoni MA, et al. Scaling system for organ specific injuries. Curr Opin Crit 1996,2:450-62.

6. Shinkawa H, Yasuhara H, Naka S, et al. Characteristic features of abdominal organ injuries associated with gastric rupture in blunt abdominal trauma. Am J Surgery 2004;187:394-7.

7. Hota PK, Babu M, Satyam G, et al. Traumatic gastric rupture following blunt trauma abdomen. Bali Med J 2014;3:49-52. 